\title{
A commentary on "Short-, mid-, and long-term incontinence outcomes in women undergoing mid-urethral sling procedures: a retrospective cohort study"
}

\author{
Ghazaleh Rostaminia ${ }^{1}$ (D) \\ Received: 29 June 2020 / Accepted: 7 July 2020 / Published online: 16 July 2020 \\ (C) The International Urogynecological Association 2020
}

This retrospective cohort study aimed to compare short-, mid-, and long-term subjective treatment success rates in women undergoing mid-urethral sling (MUS) procedures for stress urinary incontinence (SUI). The authors hypothesized that treatment success would decrease with length of follow-up. All women undergoing MUS procedures from 2001 to 2010 at a single academic institution were included in the study. For the short- and mid-term follow-up groups, an existing database was utilized addressing women undergoing MUS from 2006 to 2010. For the long-term group, patients were identified by the current procedural terminology (CPT) code for a sling procedure, and charts were reviewed for specific surgical procedures performed from 2001 to 2007. All eligible subjects were mailed questionnaires including Urogenital Distress Inventory (UDI-6), Pelvic Floor Impact Questionnaire (PFIQ-7), Patient Global Impression of Improvement (PGI-I), and Patient Satisfaction Questionnaire (PSQ). The primary outcome of treatment success was defined as responses of "not at all" or "somewhat" to both SUI subscales on the UDI-6.

Of the 896 respondents, 361 were assessed in the short term (mean follow-up time of $23.3 \pm 7.2$ months), 251 in the midterm (mean $49.8 \pm 9.1$ months), and 284 in the long term (mean $147.9 \pm 20.6$ months). The majority of MUS were retropubic slings. Treatment success was noted in $75.4 \%$ $(272 / 361)$ in the short-term group, $62.3 \%(157 / 251)$ in the mid-term group, and 67.0\% (189/284) in the long-term group $(p<0.01)$. Patient satisfaction was similar among the groups with $83 \%$ in the short-, $77 \%$ in the mid-, and $78 \%$ in the longterm groups $(p=0.31)$ reporting being "completely" or "somewhat" satisfied.

In summary, this study showed that women undergoing a primary mid-urethral sling in the mid- and long-term followup groups were less likely than their short-term counterparts to report subjective treatment success. However, after an initial decrease in treatment success from short- to mid-term followup, subjective success remained stable through long-term follow-up. The trend is consistent with that reported for the TOMUS study, which demonstrated a decrease in treatment success at 5 years compared to the initial 12-month outcomes from about $77 \%-80 \%$ to $43 \%-50 \%$ in retropubic and transobturator slings. A significant decline in the mid- and long-term MUS treatment success has now been repeatedly reported, suggesting that a gap remains in our understanding of the biomechanics of sling success and failure as well as the role of urethral support in the female continence mechanism. However, if patients are satisfied beyond 24 months they are likely to remain satisfied out to at least 10 years.

Publisher's note Springer Nature remains neutral with regard to jurisdictional claims in published maps and institutional affiliations.
Ghazaleh Rostaminia

ghazalerostaminia@yahoo.com

1 NorthShore University HealthSystem Skokie, Skokie, IL, USA 